\title{
Studies of Autoionizing States Relevant to Dielectronic Recombination
}

\section{Grant DE-FG05-85ER13394 \\ Progress Report}

\author{
prepared for \\ Department of Energy \\ Office of Basic Energy Sciences \\ Dr.J. V. Martinez
}

April, 1992

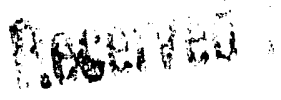

0.1902

Thomas. F. Gallagher

Department of Physics

University of Virginia

Charlottesville, VA 22901 


\section{Introduction}

In the past year we have focused most of our effort on the study of the $3 \mathrm{pnl}$ autoionizing states of $\mathrm{Mg}$. Specifically, we have measured the angular distributions of electrons ejected from these states, we have carried out theoretical analyses of the experimentally determined angular distributions, and we have also made measurements of the autoionization rates of $\mathrm{Ng} 3 \mathrm{pnl}$ Stark states in electric fields. Finally, we have recorded the spectra of the autoionizing Ba 6 pnk stark states with very high optical powers to see the $\mathrm{f}$ ine details in the wings of the spectra. Each of these activities is described in more detail in the ensuing paragiraphs.

\section{Progress}

During the past year we have analyzed the angular distributions of the electrons from the Mg 3pns states. The measurements had been done in the prior year. The analysis was done using the $K$ matrices calculated by Greene, which we had already compared to the total photoabsorption cross sectios of the $\mathrm{Mg} 3$ pns and 3 pnd $\mathrm{J}=1$ and $\mathrm{J}=3$ states. ${ }^{1,2}$ while the $\mathrm{K}$ matrix gave an excellent representation of the photoabsorption cross section, it was not clear that it would do as well for the angular distributions, for they provide a much more stringent test. However, the agreement between the experimental observations and the $K$ matrix calculations is excellent. The 
only discrepancy is that the experimental data exhibit a more pronounced dip in the parameter between the locations of the $\mathrm{Mg}^{+}$ ion lines than does the calculated $\beta$ parameter."

During the past year we have carried out the measurements of the angular distributions obtained when exciting the 3 pnd states from the bound 3 snd states. The 3 snd states are excited by two lasers linearly polarized in the same direction and are thus aligned. More specifically, they are in the $m=0$ state. The third laser, which excites these states to the 3 pnd states is also polarized in the same direction, so that the final states which can be produced are those in which $J=1$ or 3 and $m=0$. Polarizing all three lasers in the same direction ensures that only $J=1$ and 3 final states are present. Any other choice of polarization would allow the excitation of $\mathrm{J}=2$ states as well.

We measured both the total ion production and the number of electrons ejected at the angle $\theta$ relative to the polarization of the laser as we scanned the wavelength of the laser. This proceedure was repeated for ten values of the angle $\theta$ to obtain the coefficients of the angular distributions. We fit the data to the form

$$
I(\theta)=1+\beta P_{2} \cos (\theta)+\gamma P_{1}\left(\cos (\theta)+e P_{6} \cos (\theta)\right.
$$

In essence, we have measured the parameters $\beta, \gamma$, and $\epsilon$. We have used the same $K$ matrices provided by Greeene to 
calculate the total $J=1$ and $J=3$ absorption to calculate the angular distributions. In addition, Robicheaux and Greene have calculated the angular distributions using an expanded $\mathrm{K}$ matrix and a different form of the theory. Both calculations give results which are in excellent agreement with the experimental results.

In the past we have studied the autoionizing Ba 6 pnk Stark states, and we have found that from a single bound 6snk stark state that we excite a single 6 pnk stark stste! The widths of the lines could be fit easily to Lorentzians and the dependence was easily explained using a hydrogenic description of the stark effect. The previous measurements did not address the question of what the channels are in the presence of an electric field. During the past year we have examined the wings of the bisnk-bpnk transitions, using high optical powers, to address this question. We have observed clear structure in the wings due to the other Stark states. Typically, they produce a modulation of $15 \%$ of the total intensity. While we can easily identify the probable source of the modulation, we have not yet succeeded in carrying through a complete analysis. An extension of the quantum defect theory of the Stark effect, as worked out by Harmin or Sakimoto,,$^{5.6}$ is required.

Finally, we have observed the Mg 3 snk-3pnk transitions in electric fields. The results are not at all what we expected to see. Only in rare cases does the spectrum reduce to one line as 
it does in Ba. Usually there are half a dozen strong lines. Why $\mathrm{Mg}$ is so different is not immediately clear, but we suspect that the difference stems from the fact that in $M g$ the $f$ ine rtructure splitting of the $3 p$ state of the ion is only $92 \mathrm{~cm}^{-1}$, so that the Stark states of $n=10$ to 20 are mixtures of states converging to both limits, with the result that the bound and autoionizing Stark states are not the same. We plan to use a microwave field as well, to mimic the effect of the time varying microfields in a plasma, to see if the results in that case are also different from the $\mathrm{Ba}$ results. 

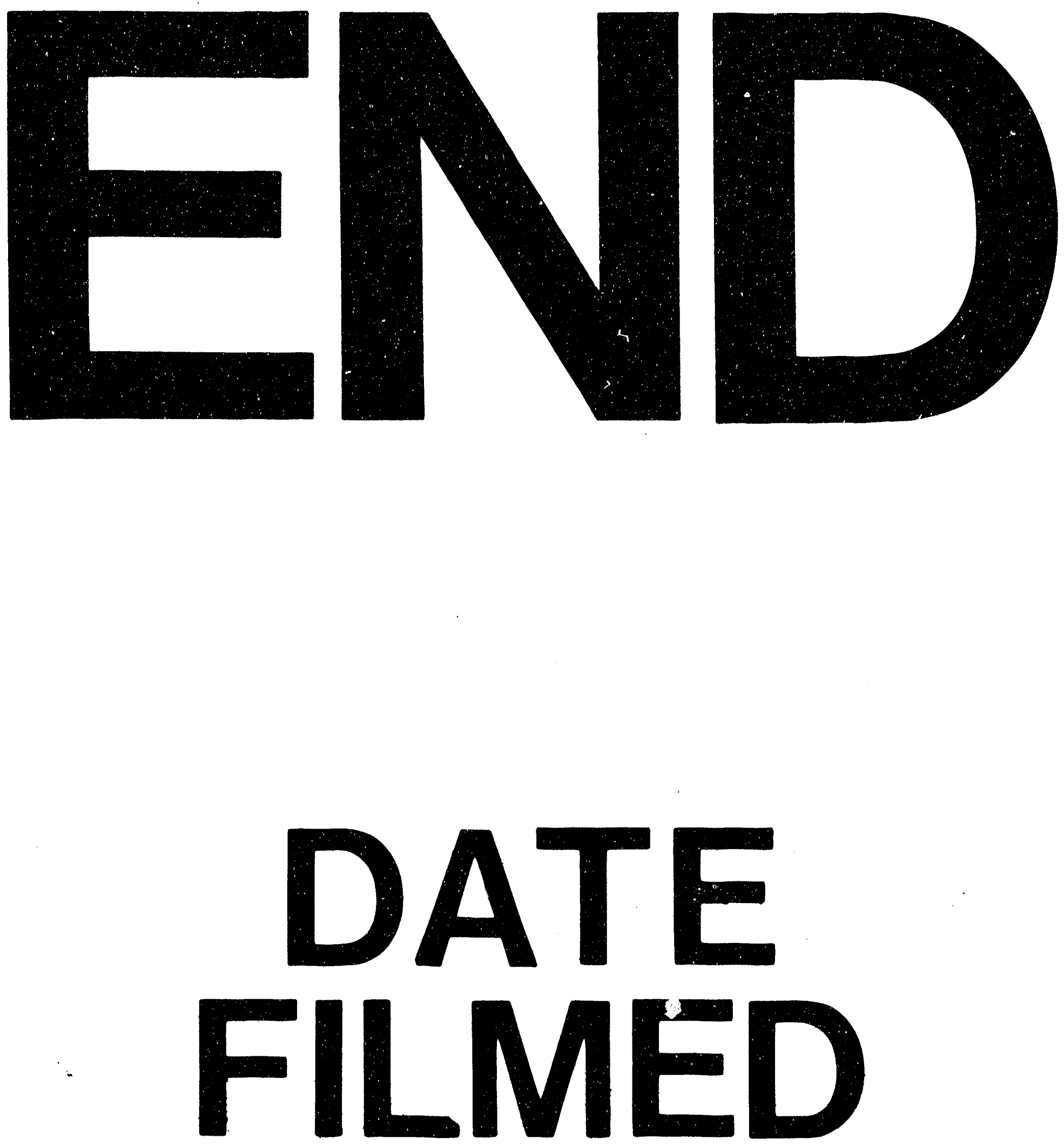

1

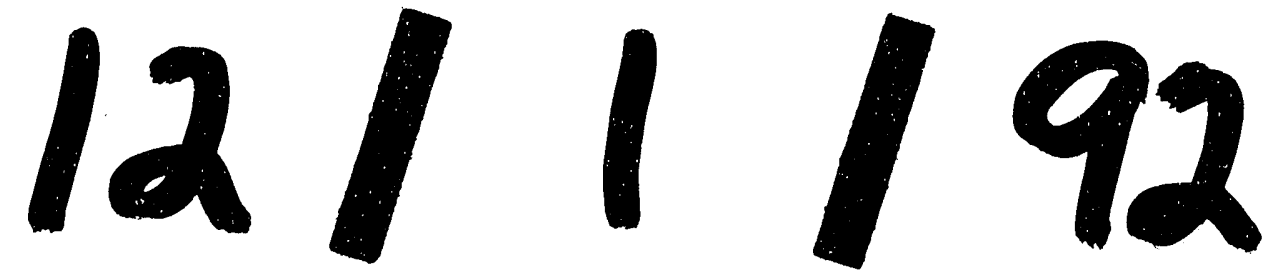


